\title{
Enhanced Engine Performance during Emergency Operation using a Model-Based Engine Control Architecture
}

\author{
Jeffrey T. Csank ${ }^{1}$ and Joseph W. Connolly ${ }^{2}$ \\ NASA Glenn Research Center, Cleveland, OH, 44135, USA
}

\begin{abstract}
This paper discusses the design and application of model-based engine control (MBEC) for use during emergency operation of the aircraft. The MBEC methodology is applied to the Commercial Modular Aero-Propulsion System Simulation 40k (CMAPSS40k) and features an optimal tuner Kalman Filter (OTKF) to estimate unmeasured engine parameters, which can then be used for control. During an emergency scenario, normally-conservative engine operating limits may be relaxed to increase the performance of the engine and overall survivability of the aircraft; this comes at the cost of additional risk of an engine failure. The MBEC architecture offers the advantage of estimating key engine parameters that are not directly measureable. Estimating the unknown parameters allows for tighter control over these parameters, and on the level of risk the engine will operate at. This will allow the engine to achieve better performance than possible when operating to more conservative limits on a related, measurable parameter.
\end{abstract}

\section{Nomenclature}

$\begin{array}{ll}\text { CMAPSS40k } & \text { Commercial Modular Aero-Propulsion System Simulation 40k } \\ \text { EEC } & \text { Enhanced engine controller } \\ E P R & \text { Engine Pressure Ratio } \\ \text { Fnet } & \text { Thrust, lbf } \\ \text { HPC } & \text { High pressure compressor } \\ H P C S M & \text { High pressure compressor surge margin, \% } \\ \text { IWP } & \text { Integral wind-up protection } \\ \text { LPC } & \text { Low pressure compressor } \\ \text { LPT } & \text { Low pressure turbine } \\ \text { MBEC } & \text { Model based engine control } \\ N c & \text { Core speed, rpm } \\ N f & \text { Fan speed, rpm } \\ \text { OTKF } & \text { Optimal tuner Kalman filter } \\ P a & \text { Ambient pressure, psi } \\ P C & \text { Power code, \% } \\ \text { PI } & \text { Proportional integral controller } \\ P L A & \text { Power lever angle, degrees } \\ P R \text { HPC } & \text { Pressure ratio across high pressure compressor } \\ P 2 & \text { Inlet pressure, psi } \\ P 25 & \text { Low pressure compressor exit pressure, psi } \\ P s 3 & \text { Combustor static pressure, psi } \\ P 50 & \text { Low pressure turbine exit temperature, degrees Rankin } \\ \text { RamP } & \text { Ram pressure } \\ \text { SLS } & \text { Sea level static } \\ T 25 & \text { Low pressure compressor exit temperature, degrees Rankin } \\ T 30 & \text { High pressure compressor exit temperature, degrees Rankin } \\ T 40 & \text { High pressure turbine inlet temperature, degrees Rankin } \\ \end{array}$

\footnotetext{
${ }^{1}$ Aerospace Engineer, Intelligent Controls and Autonomy Branch, jeffrey.t.csank@nasa.gov, AIAA Member ${ }^{2}$ Aerospace Engineer, Intelligent Controls and Autonomy Branch, joseph.w.connolly@nasa.gov, AIAA Senior Member
} 
High pressure turbine exit temperature, degrees Rankin

Low pressure turbine exit temperature, degrees Rankin

Variable bleed valve

Variable stator vane, degrees

Fuel flow rate, $l b / s$

High pressure compressor flow, $l b / s$

Change in fuel flow, $l b / s$

\section{Introduction}

$\mathrm{T}$ HE National Aeronautics and Space Administration Aeronautics Research Mission Directorate is focused on providing solutions to improve aviation safety and reduce the environmental impacts of air transportation, amongst other objectives. Specifically of interest, in relation to aviation safety, is the ability to use an aircraft's engines to help pilots avert or recover from emergency situations. ${ }^{1}$ The focus is on two general types of emergency scenarios: 1) runway incursion, and 2) rudder/tail failures. These emergency scenarios are derived from events of actual in-flight airframe malfunction or damage, where flight crews resorted to engine throttle modulation to maintain aircraft control. $^{2,3}$ The runway incursion scenario focuses on situations where an aircraft's available takeoff distance is suddenly decreased. In this event, the availability of additional contingency thrust may enable safe takeoff in a shortened distance by allowing the aircraft to reach its safe takeoff speed sooner, thus avoiding the disaster. To provide this additional thrust, a control mode referred to as overthrust has been previously proposed. ${ }^{1}$

The rudder/tail failure scenario encompasses cases where problems with the flight control surfaces are encountered, such as stuck or broken actuators, airframe damage, and damaged or missing controls surfaces. Studies have shown that, although it is possible to land an aircraft using only the throttles to maneuver the aircraft, this is an extremely difficult task due to the slow engine response times and the inability to damp out phugoid and dutch roll modes. $^{4,5}$ In this situation, increasing the responsiveness of the engines in order to counter these modes is the primary goal; this is done through the faster engine response control mode. One approach that has been developed for designing a fast engine response controller involves modifying the engine's actuators to increase the performance near idle speeds, a control solution known as high speed idle; this has been shown to decrease the time to reach full power. ${ }^{6}$

Past studies have investigated the ability to improve engine performance during emergency scenarios by altering the control algorithm and intelligently reducing the safety limits., ${ }^{2,3}$ In these studies, the risk of an engine failure is assumed to be dependent on the engine's rotor speeds, pressures, and temperatures. Therefore, the engine limits (speeds, pressures, and temperatures) can be raised to higher levels based on the level of risk that is deemed acceptable during an emergency situation, resulting either in additional steady-state (overthrust) or transient (faster engine response) performance. A risk boundary concept has been proposed, where the engine parameters that correspond to a predetermined maximum risk of failure are determined off-line. ${ }^{3}$ The pilot/airframe can then request the desired thrust up to this predetermined risk level.

The goal of model based engine control (MBEC) is to provide tighter control of critical unmeasured gas path parameters, through advanced estimation techniques, and to improve the overall performance of the gas turbine. ${ }^{8}$ Currently, commercial aircraft engines are designed to meet cautious end-of-life safety margins regulated by the Federal Aviation Administration. These safety margins limit engine parameters, both measured and unmeasured, such as rotational speeds, temperatures, pressures, and stall margins, which sets specific risks for various types of engine component failures. MBEC will allow for operation of the engine with less-conservative margins, as an on-board model can provide a more accurate margin estimate at the actual condition of the engine. Rather than operate under a margin designed for end-of-life, as currently done, a limit based on this estimated engine condition may be used, improving performance, especially in a new engine. For example, a surge margin limiter can be developed to ensure that a lower surge margin threshold can be used during transient changes, resulting in a faster response.

The approach proposed here relies on the integration of the MBEC architecture with the risk boundary concept to safely provide an enhanced engine response during an emergency scenario, through a simple and intuitive closed-loop control solution. The complex schemes currently used for assessing the risk and changing the controller during an emergency scenario can be replaced with designs which take advantage of the estimated parameters of interest, provided by an on-board model, and directly use them in the control logic.

As was done previously, the Commercial Modular Aero-Propulsion System Simulation 40,000 (CMAPSS40k) ${ }^{9,10}$ will be used as the baseline commercial-type turbofan engine simulation for investigating methods of achieving additional engine performance. Advanced control algorithms considered in previous studies have included sliding mode controllers ${ }^{11}$ and L1 adaptive control theory, ${ }^{12}$ with attempts to minimize changes in the traditional engine control architecture. This paper builds on the previous MBEC work performed by the authors. Section II discusses 
the MBEC architecture while Section III discusses the implementation of MBEC with a newer version of CMAPSS40k than the previous MBEC work. Section IV discusses changes made to MBEC controller for the emergency situations, which are mainly related to the limiters in the protection logic; simulation results of the MBEC system with enhanced performance are shown in Section V and summarized in Section VI.

\section{The Model-Based Approach}

Traditional commercial aircraft engine control architectures rely on measured data to provide the aircraft with the requested power (thrust) and ensure the engine does not exceed any of its physical (pressures, temperatures, rotor speeds) or operational (surge margin) limits. Some key engine parameters are not measured, such as thrust, highpressure turbine inlet temperature, and compressor surge margin, so representative parameters are used instead. For example, since thrust is not measured in flight, current commercial aircraft engines regulate the fan speed $\left(N_{f}\right)$ or engine pressure ratio $(E P R)$, both of which have a direct relationship with thrust. ${ }^{13,9}$ Similarly, since surge margin is not measurable, to avoid a high-pressure compressor (HPC) surge, an acceleration limiter is implemented to limit the acceleration of the core, or high-pressure, spool or the ratio of fuel flow (Wf) to combustor static pressure (Ps3), commonly noted at $W f / P s 3$. As the engine degrades, the assumed relationship between the controlled engine outputs and the unmeasured engine parameters tends to break down, leading to degraded performance and operability.

MBEC relies on an on-board engine model, which can provide a more accurate estimate of the desired engine parameters and potentially increase the performance of the engine. Improved estimation of thrust allows the engine to deliver more consistent power based on the throttle setting. Replacing the current techniques for limiting acceleration with a controller regulating the estimated surge margin can impact the dynamic response of the engine for large accelerations. Estimating the surge margin and other operating margins with relative accuracy has an additional benefit in that the amount of margin required to ensure that the limit is not violated during worst case conditions may be decreased.

The MBEC architecture is comprised of three main components, as illustrated in Figure 1: an engine, or "truth," model, an on-board model design to provide real-time estimates of desired unmeasured parameters, and a controller with limit protection logic. The individual controllers are designed as simple proportional integral (PI) controllers, which is consistent with traditional control architectures. The protection logic uses a minimum (min)/maximum (max) selector to provide an appropriate fuel flow command to the actuator. For the simulation study presented in this paper, CMAPSS40k ${ }^{9}$ will serve as the engine model, an optimal tuner Kalman Filter (OTKF) ${ }^{14}$ will serve as the on-board model providing estimates of thrust, high pressure turbine inlet temperature, and stall margin, and the controller is modified from the standard controller in CMAPSS40k to use the estimated measurements.

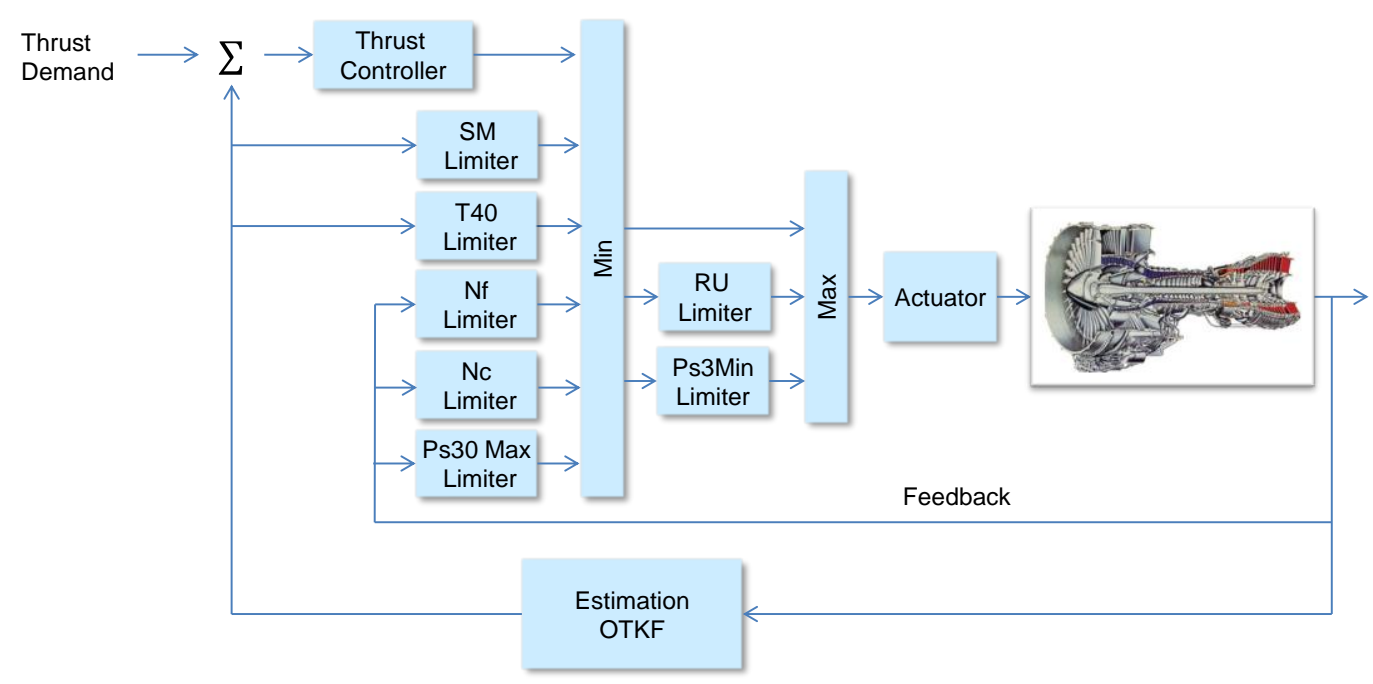

Figure 1 MBEC Control Architecture. The thrust controller, T40 Limiter, and SM Limiter all rely on estimated measurements generated from the Optimal Tuner Kalman Filter. 


\section{Implementation of MBEC to CMAPSS40k Simulation}

CMAPSS40k is a nonlinear, physics-based, component-level dynamic engine model with a realistic closed-loop controller written in the MATLAB/Simulink environment. ${ }^{9,10}$ CMAPSS40k models a 40,000-pound thrust class, highbypass, dual-spool turbofan engine. The low-pressure components (fan, compressor (LPC), and turbine (LPT)) are connected by the fan shaft, and the high-pressure components (HPC and turbine (HPT)) are connected by the core shaft (which rotates at a speed $N c$ ). The fan, compressors, and turbines are modeled using performance maps that relate the pressure ratio, mass flow rate, and corrected speed for each component. CMAPSS40k also contains scheduled variable stator vanes $(V S V)$ and a variable bleed valve $(V B V)$. The engine model includes a typical turbofan engine sensor suite, providing measurements of $N f, N c$, the exit pressures and temperatures of the LPC (P25 and T25) and LPT (P50 and T50), Ps3, and the exit temperature of the HPC (T30). CMAPSS40k allows for modeling degradation of the engine following a fleet average profile of engine deterioration. By modifying the efficiency and flow capacity health parameter inputs for each component - fan, LPC, HPC, HPT, and LPT - various engine life conditions maybe simulated. The traditional control architecture consists of a set point controller, which can regulate EPR or $N_{f}$, max limiters, and min limiters.

A simplified block diagram of the traditional closed-loop architecture is shown in Figure 2. The max limiters impose constraints on $N f, N c, P s 3, T 50$, and include an acceleration schedule to protect against HPC surge. The min limiters include $P s 3$ and a ratio unit $(R U)$ limiter to protect against surge during a deceleration by regulating $W f / P s 3$. The command to the fuel flow actuator (referred to as Actuator in Figure 2), $W f$, is determined by computing the min of the max limiters and the set point controller and then the max of the resulting signal and the min limiters. The reader is referred to Ref. 10 for more on the standard CMAPSS40k controller. The EPR and $N f$ controllers utilize a gain scheduled PI controller along with integral windup protection.

The controller used in this study has been improved over that released with CMAPSS40k through the use of a new gain scheduling scheme. Instead of using altitude and Mach number to schedule gains, the controller uses ambient pressure $(P a)$ and ram pressure $(\operatorname{Ram} P)$, the total pressure at the inlet face $(P 2)$ divided by $P a$. The throttle mapping, which relates the power lever angle ( $P L A)$ to thrust via $E P R$ or $N f$, also has been updated to increase max thrust at sea level static (SLS) (0 ft altitude and Mach 0.0) from 34504.9 to 40089.6 pounds-force. Note that, for comparison purposes, the thrust schedule for the baseline version was also updated and the model was re-executed using the new thrust values. The maximum and minimum limit values were also modified for this newer version, but the original limit values were used here since they correspond to known risk levels.

To implement MBEC, the standard engine control architecture shown in Figure 2 is replaced by the MBEC control architecture of Figure $1,{ }^{10}$ where the estimated signals produced by the OTKF rely on the standard set of engine outputs from the CMAPSS40k engine. (Note that previous MBEC work in Ref. 8 did not include the P25 and T25 measurements; when used, these measurements increase the accuracy of the estimated parameters.) For this application there are 8 sensors and 10 health parameters, which define an underdetermined estimation problem that is solvable using the proposed OTKF approach. The on-board model is a piece-wise linear model of CMAPSS40k and consists of a steady-state trim model used to create a delta (the difference between the output of the steady-state model and the actual feedback), and a state space model. The state space matrices are calculated for 7 altitudes, 9 Mach

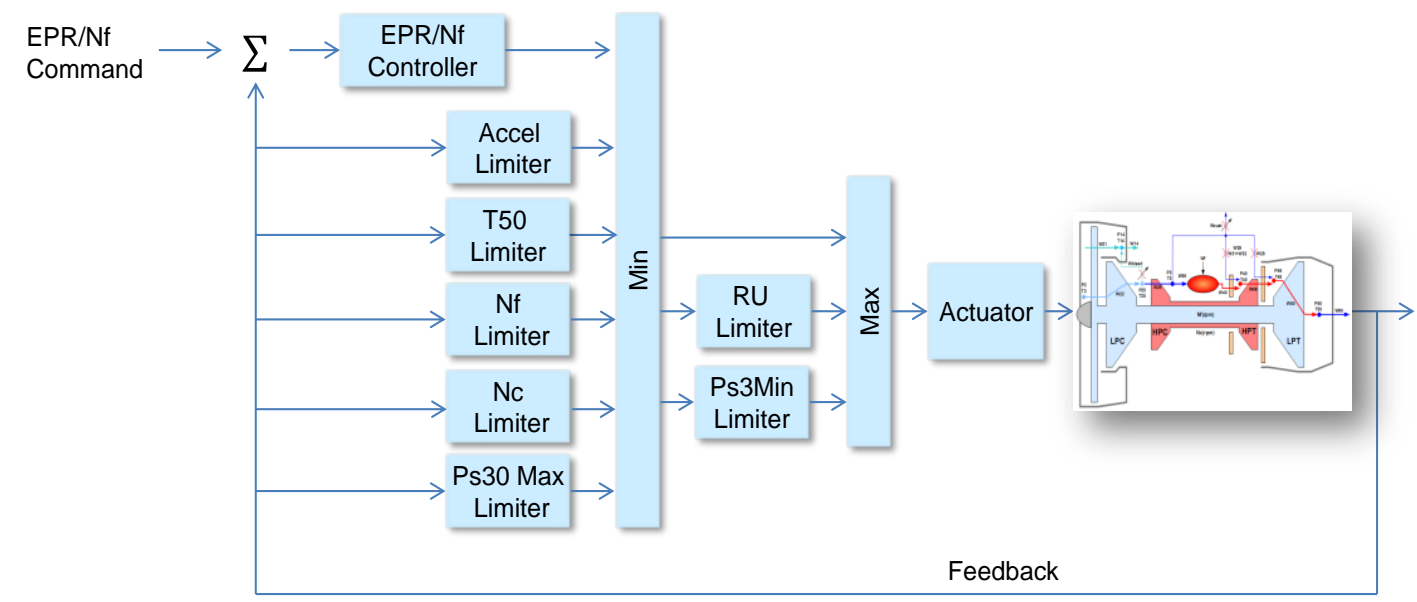

Figure 2 CMAPSS40k Traditional Control Architecture

4

American Institute of Aeronautics and Astronautics 
numbers, and 14 fan speeds, while the trim point model uses a much finer grid (21 altitudes, 18 Mach numbers and 64 fan speeds). The inputs to the estimation OTKF include $W f, V S V$, and $V B V$. The estimated parameters from the OTKF are the combustor exit temperature (T40), thrust (Fnet), HPC SM, LPC SM, HPC flow ( $W_{H P C}$ ), and the pressure ratio across the HPC $\left(P R_{H P C}\right)$. The latter parameters, $W_{H P C}$ and $P R_{H P C}$, will be used to improve the estimation of the HPC surge margin, especially during engine transients. The nonlinear equation for these estimations is:

$$
H P C S M=\frac{P R_{\text {surge }}\left(W_{H P C}\right) d V S V(V S V, N c)-P R_{H P C}}{P R_{H P C}} \times 100
$$

where $d V S V$ is the difference between $V S V$ input and the ideal $V S V$ angle, which is a function of $V S V$ and $N c$.

The standard EPR/N $f$ controller is replaced with a thrust controller, which regulates the estimated thrust from the OTKF. This controller is a gain scheduled PI controller with integral windup protection and has the control law:

$$
u=K p_{F c} K p_{F n t} e+\int K i_{F c} K i_{F n t}\left(e-I W P\left(\Delta u_{W f}\right)\right)
$$

where $K p_{F c}$ and $K i_{F c}$ are gains scheduled on the actual $R a m P$ and $P a, K p_{F n t}$ and $K i_{F n t}$ are gains scheduled on the feedback values of $P a$ and Fnet, and $e$ is the tracking error of Fnet. Integral windup compensation includes a gain, $I W P$, and $\Delta u_{W f}$ is the difference between the fuel flow from the thrust controller and the fuel flow to the engine, as determined at the previous time step; this is the same approach used by CMAPSS40k. The PI controllers were scheduled to meet a bandwidth of $0.75 \mathrm{~Hz}$ and phase margin of 60 degrees at 9 ambient pressures, 5 ram pressures, and 12 thrust values ranging from 4,000 lbf to 40,000 lbf.

Other modifications to the control system were required for MBEC to work correctly. A set point function was created to convert the current throttle position to the demanded thrust. The relationship between throttle position and thrust is defined at five different $\operatorname{Ram} P(0.9995,1.0280,1.1163,1.2752$, and 1.5240). At each $\operatorname{RamP}$, several altitudes (or ambient pressures) were used to verify this relationship and remove any outlier data points. The acceleration limiter was modified to regulate the surge margin estimate instead of limiting the core acceleration based on the corrected core speed. The surge margin limiter consists of a gain scheduled PI controller, with integral windup protection. The $T 50$ limiter was replaced with a T40 limiter, which also includes a PI controller with integral windup protection.

\section{Implementation for Emergency Control Work}

MBEC has previously been investigated as a means to increase the fuel efficiency of a high bypass turbofan engine. This paper intends to demonstrate that the MBEC concept has additional benefit regarding the enhancement of the engine performance during emergency flight scenarios. The proposed control modes of interest are in response to two emergency flight scenarios: i) overthrust control mode for a runway incursion scenario, and ii) faster engine response for a rudder/tail failure. These control modes are discussed below in more detail.

\section{A. Overthrust}

The overthrust control mode was designed in response to a runway incursion-type event, where the available takeoff distance is suddenly decreased. In this event, additional thrust produced from the engine can help the aircraft reach its safe takeoff speed over a shorter distance, potentially avoiding the disaster. For the engine to produce this additional thrust, it must operate at elevated pressures, temperatures, rotor speeds, and, consequently, an elevated risk of engine failure. Some of the research regarding overthrust has addressed the evaluation of risks associated with implementing and operating at these performance levels ${ }^{7}$, the development of a risk management architecture to extend engine limits based on assessment of the emergency severity ${ }^{2}$, and evaluating the change in risk and performance. ${ }^{3,6}$

One solution to the overthrust problem includes defining, off-line, values for the engine parameters that correspond to a predetermined maximum risk of failure, extending all the engine limiters to that value, and allowing the pilot/airframe to request the desired thrust up to this point. ${ }^{3}$ The maximum allowable probability of an engine failure accounts for various failures, such as turbine blade failure or rotor disk failure, and is determined by analyzing the rotor speeds and turbine temperatures using an available MATLAB® / Simulink® based tool. ${ }^{7}$ The main result of this approach is calculation of a boundary function representing a constant level of failure probability. Here, this risk function operates on five inputs ( $N f, N c, T 40$, HPT exit temperature (T48), and T50), defining a five-dimensional surface on which the probability of failure remains constant.

To simplify implementation of this approach, the risk function was analyzed by looking at disk failure and blade failures separately to determine if the number of inputs could be reduced. It was found that the risk was sensitive to $N c$ but not $N f$; therefore $N f$ could be removed without significant impact. Because the failure rate was sensitive to all three of the temperatures, a linear least-squares fit was developed that correlated T40 to T50, T40 to T48, and T48 
to $T 50$, allowing the three temperatures to be expressed as a single value. With a good correlation between these temperatures, a single temperature limit could be constructed.

An example application of this predesigned risk boundary concept is provided in Ref. 3 through simulation of CMAPSS40k at various takeoff conditions. It was determined that the CMAPSS40k engine operates nominally with an engine failure rate between $10^{-7}$ and $10^{-5}$. Data was gathered to determine the acceptable engine limits corresponding to a failure rate of $10^{-3}$. The engine controller was modified by replacing the T50 limiter with the T40 limiter and the acceleration schedule with a surge margin controller, and adding logic that allows the engine to operate at the elevated risk and power levels.

In this paper, the risk boundary approach is integrated with MBEC as shown in Figure 3. For the enhanced engine controller (EEC), the PLA input is converted to a Power Code $(P C)$ variable that ranges from 0 to 100 in normal operation or 0 to 110 during an emergency. For a $P C$ from 0 to 100 , the fuel flow signal is determined based on the thrust controller and standard limiters, with the exception of the appropriate temperature limiter; a limiter on $T 50$ was used for the standard controller and one on $T 40$ for MBEC. For a $P C$ from 100 to 110, the fuel flow signal is combined with the amount of fuel flow required to reach the $10^{-3}$ risk level based on the elevated temperature and $N c$ limits. Note that in Ref. 3 there is discussion regarding how the Ps3 limiter affects the performance during enhanced operation, along with results from simulations with and without the $P s 3$ limiter. For this paper, all the results assume that the Ps 3 limiter is disabled since the effect of enabling this limit is the same for MBEC.

\section{B. Faster Engine Response}

The faster engine response control mode is designed to respond to a rudder/tail failure flight scenario in which there is a problem with the flight control surfaces. The primary purpose is to increase the dynamic response of the engine. As previously mentioned, there have been several attempts at increasing the responsiveness of the engine, which share some common features. ${ }^{2,3}$ Each attempt has considered increasing the bandwidth of the EPR or $N f$ controller to allow the engine to respond faster to the throttle command, mainly for small transients where no limiters are activated. Another common feature is modification of the acceleration schedule, or acceleration limiter, to allow the engine to respond faster to large throttle transients by allowing the HPC to operate closer to the surge line. (In an emergency scenario, this reduction in the surge margin limit may be acceptable.) The difference between each method is in how the acceleration schedule is modified. One method uses a lookup table model to determine the offset added to the schedule based on the amount of risk deemed acceptable at the current time. ${ }^{2}$ This method is difficult to implement as the lookup table model required for high accuracy is quite large, especially when accounting for different levels of risk. Another method determines the surge margin limits that correlate to a specific risk, similar to the overthrust procedures. This method provides much more consistent results and is easier to implement. ${ }^{3}$

The success of all these methods relies on the ability to estimate the current engine health, or life, without the use of an on-board model, and the assumption that the engine will degrade similar to the fleet average. The integrated OTKF and MBEC approach offers the advantage of estimating the engine health in real-time and providing a surge

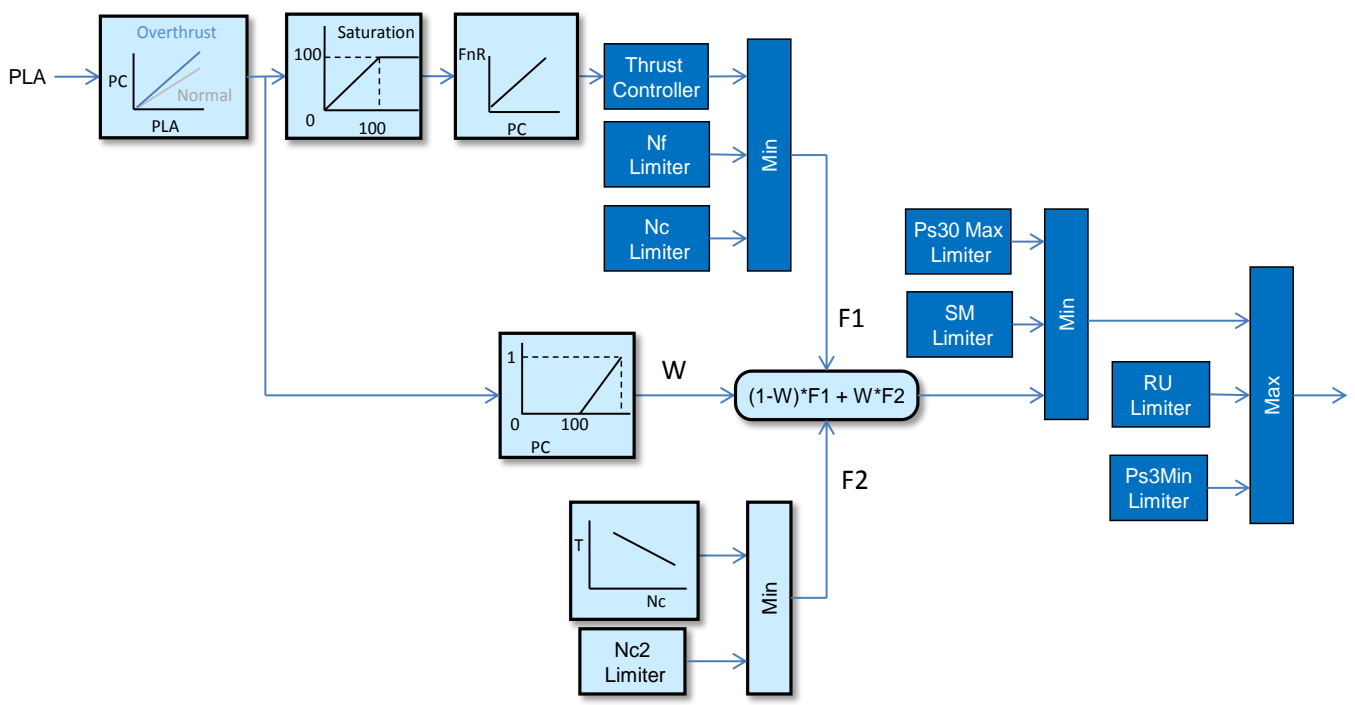

Figure 3 EEC architecture. Lightly shaded blocks are modifications from the standard control architecture. Note that feedback paths were removed for simplification. 
margin estimate based on current engine parameters. To request additional performance in the MBEC architecture, the surge margin limit would simply be reduced to a value determined from an off-line risk assessment similar to the method shown by Liu, et.al. ${ }^{3}$ The surge margin limiter would allow the estimate to reach the newly specified surge margin limit.

\section{Results}

The MBEC architecture, shown in Figure 1, is implemented with CMAPSS40k. A simulation study is conducted comparing the nominal MBEC (no engine enhancements) configuration to CMAPSS40k using a chop and burst throttle input at both takeoff and cruise conditions. This particular throttle profile provides the information necessary to evaluate both the accuracy of each method and the transient performance. Accuracy is evaluated by the ability of the closed-loop controller to deliver the requested thrust, while the transient performance is evaluated through the relationship between the response time, or settling time, and minimum HPC SM.

Figure 4 compares the thrust (top) and EPR (bottom) produced from the engine using the standard CMAPSS40k controller and the MBEC architecture. While both controllers drive EPR to the demand, the MBEC is better at achieving the requested thrust. The difference between the CMAPSS40k thrust output and the demanded thrust indicates the difficulty in modeling the relationship between $E P R$ and thrust. The difference between the MBEC thrust output and thrust demand demonstrates the accuracy of the OTKF and linear model and suggests that the tracking error can be attributed to modeling errors, slight biases in the engine outputs used to create the estimate, etc. The results show one advantage of the MBEC architecture: with an accurate estimate of thrust, the MBEC controller can drive the actual thrust of the engine closer to the intended demanded thrust than with a controller tracking either EPR or $N_{f}$.

A similar simulation study using a chop and burst throttle profile (like that shown in Figure 4) is also performed to test the dynamic performance and steady-state accuracy of the MBEC approach. This study considers the transitions between 500 random takeoff and 500 random cruise conditions. Takeoff conditions are defined on an altitude range of 0 to 5,500 ft and from 0.0 to $0.25 \mathrm{Mach}$. The throttle profile transitions from a climb/takeoff setting (PLA 72 to 80 ) to idle (PLA 44 to 48 ) and back to takeoff (PLA 76-80). Cruise conditions are within a range of 18,000 to 36,000 feet and 0.4 to 0.8 Mach. The throttle profile transitions from cruise/climb setting (PLA 68-76) to flight idle (PLA 4650 ), and back to cruise (68-74). Any steady-state operating point at which any steady-state limiter (such as max $N c$, min $P s 3$, etc.) is active, for either controller, will be omitted since the error between the thrust produced and demanded is due to an engine limitation rather than system (modeling) error. The overall steady-state accuracy for both takeoff and cruise are shown in Table 1. Of interest is the number of tests in which the steady-state thrust is within $\pm 100 \mathrm{lbf}$ and $\pm 250 \mathrm{lbf}$ of the actual commanded thrust. At takeoff, the MBEC controller can drive the engine to the desired
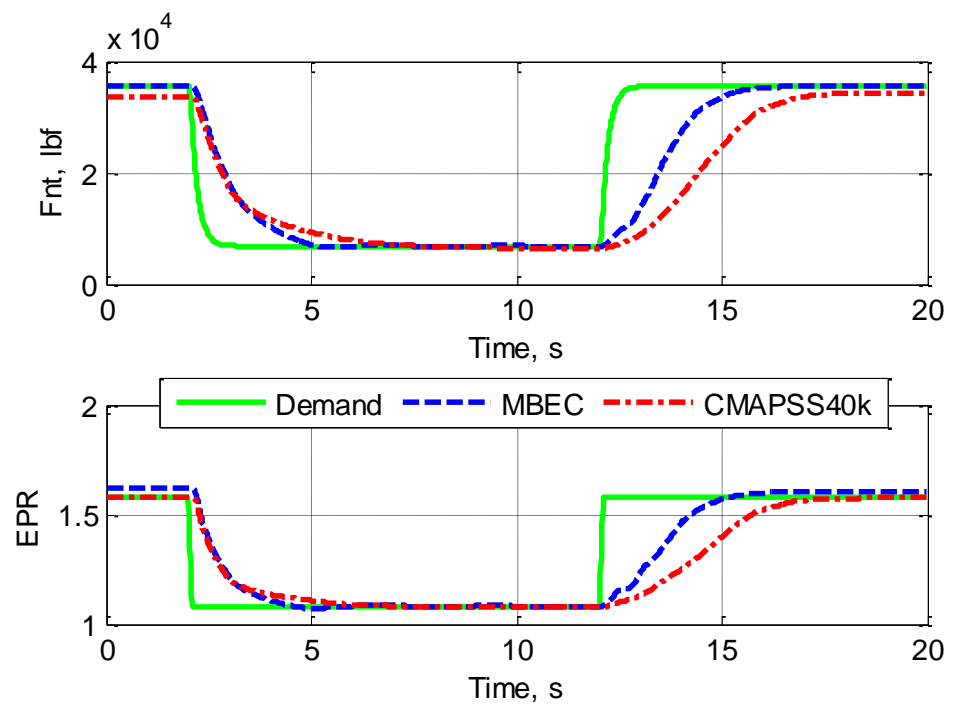

Figure 4 Comparison between the standard CMAPSS40k controller and MBEC at an altitude of 885.6ft and 0.1081 Mach. The top plot compares the thrust response compares the demanded and the bottom compares EPR. 
thrust value, to within $\pm 100 \mathrm{lbf}$, in $99.4 \%$ of the tested conditions. Compared to the traditional approach (11.3\% of the time), this is a great improvement.

From the burst portion of the throttle profile, the dynamic performance of the closed-loop system is determined and compared in Table 2. The CMAPSS40k controller relies on an acceleration schedule to protect the compressor from reaching/exceeding the surge line, which limits the core acceleration and is developed off-line for an average engine. Therefore, some engines will be more likely to surge while other engines are more capable of achieving better performance. The engines that are capable of achieving better performance are limited due to the overly conservative limiter required for those engines that perform worse than average. The MBEC approach relies on directly limiting surge margin based on an estimated value, which can allow all engines to reach to a predetermined limit, maximizing performance.

Figure 5 shows how the relationship between the settling time and minimum HPC surge margin varies at each test case for an engine controlled with the standard CMAPSS40k controller and MBEC. For takeoff conditions, the goal is to be able to transition from an idle setting to a takeoff setting in less than 5 seconds,

Table 2 Comparison of the average dynamic performance for the CMAPSS40k baseline control and MBEC for 500 random takeoff flight conditions.

\begin{tabular}{l|c|c}
\hline \hline & CMAPSS40k & MBEC \\
\hline \hline Rise Time (s) & 2.920 & 2.127 \\
\hline Rise Time Standard Deviation (s) & 0.3027 & 0.1720 \\
\hline Settling Time (s) & 5.392 & 5.008 \\
\hline Min Surge Margin (\%) & 13.51 & 14.11 \\
\hline Min Surge Margin Standard Deviation (\%) & 1.0734 & 1.6132 \\
\hline
\end{tabular}

which meets Federal Aviation Administration transient requirements. ${ }^{15}$ The surge margin controllers are designed for a minimum surge margin of $12 \%$. The results demonstrate that MBEC provides a more consistent settling time across the profiles considered, with a less-consistent minimum surge margin. For CMAPSS40k, 42.5\% of the test cases had a settling time greater than 5 seconds, and $9.5 \%$ with a surge margin less than $12 \%$. For MBEC, $10.20 \%$ of the accelerations took longer than 5 seconds and $10.20 \%$ had a surge margin less than $12 \%$. The MBEC surge margin controller was tuned at only a few different conditions for a throttle step change to meet the 5 second thrust transient

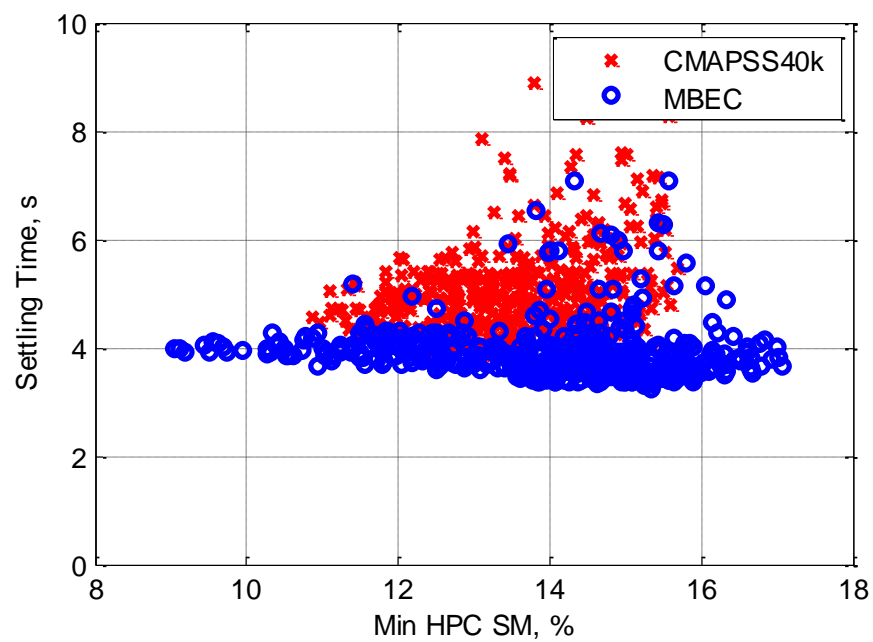

Figure 5. Comparison of the relationship between the settling time and minimum surge margin for CMAPSS40k and MBEC controlled engines. 
requirement and preserve a particular surge margin (12\%). Higher fidelity surge margin models, more complex surge margin controllers, and tuning at additional flight conditions could improve the performance of the controller.

\section{A. Overthrust}

Previous work ${ }^{3}$ described the concept of a risk-based control mode intended to increase maximum engine thrust during an emergency scenario (e.g., runway incursion). When active, the so-called overthrust control mode increases thrust output until the calculated failure probability reaches a preset value (in this case, $10^{-3}$ failures per flight hour). Unfortunately, the sensor suite within CMAPSS40k does not include $T 40$ or $T 48$, so the previous work designed the control mode to use T50 data to infer T40 and T48 values; when tested at different operating conditions, this resulted in a spread of the risk values around the desired $10^{-3}$ threshold.

The MBEC controller is modified to resemble the architecture for enhanced engine performance ${ }^{3}$ as shown in Figure 3 and tested to demonstrate the advantage of MBEC, particularly for emergency scenarios. Instead of using the measured $T 50$ values, the estimated values of $T 40$ from the MBEC architecture are utilized. (Recall that the MBEC architecture developed in this work uses a newer version of CMAPSS40k than the enhanced engine performance work.) In an attempt to account for the different power management controllers, the engine limiter values found in the previous version of CMAPSS40k will be used for $\max N f$, $\max N c$, and $\max P s 3$. The $T 50$ max will be set to $1490^{\circ} \mathrm{R}$, which was assumed in the baseline risk calculations. These two overthrust methods, using measured T50 or estimated T40 values, were tested at 200 random low-altitude (0-5500 feet), low-Mach number (0-0.25) conditions, spanning the full life-cycle of the engine (50-hour to end-of-life). Note that the case number is consistent throughout, however the flights are not numbered in a meaningful fashion. Although Figure 6 shows that both methods produce similar percent increases in maximum thrust, with the use of the traditional T50 sensor performing slightly better, the results in Figure 7 and Figure 8 demonstrate the clear advantages of using MBEC.

Figure 7 plots the failure probability for the 200 test cases when the engine operates at baseline maximum thrust, with overthrust using T50 sensor, and with overthrust using MBEC (estimated T40). As expected, the baseline risk remains below the FAA-dictated allowable failure probability of $10^{-5}$ per flight hour in $99.5 \%$ of the tests cases. Using the $T 50$ sensor, the failure probabilities are not constrained to the elevated $10^{-3}$ level and exhibit a relatively large spread. The risk function is highly sensitive to the hotter HPT temperatures, and the correlation dataset used to infer T48 and T40 from T50 is insufficient. On the other hand, using MBEC allows for the direct use of T40 in the overthrust control algorithms, producing results tightly grouped around $10^{-3}$.

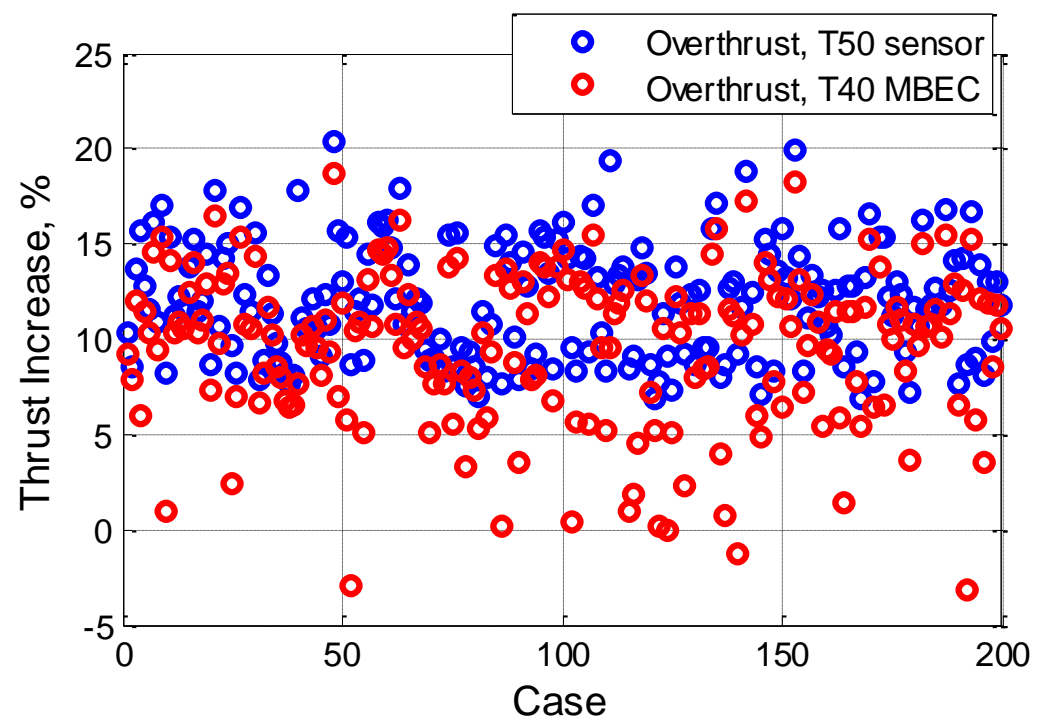

Figure 6 Net thrust improvements with MBEC and enhanced engine operation control architecture 


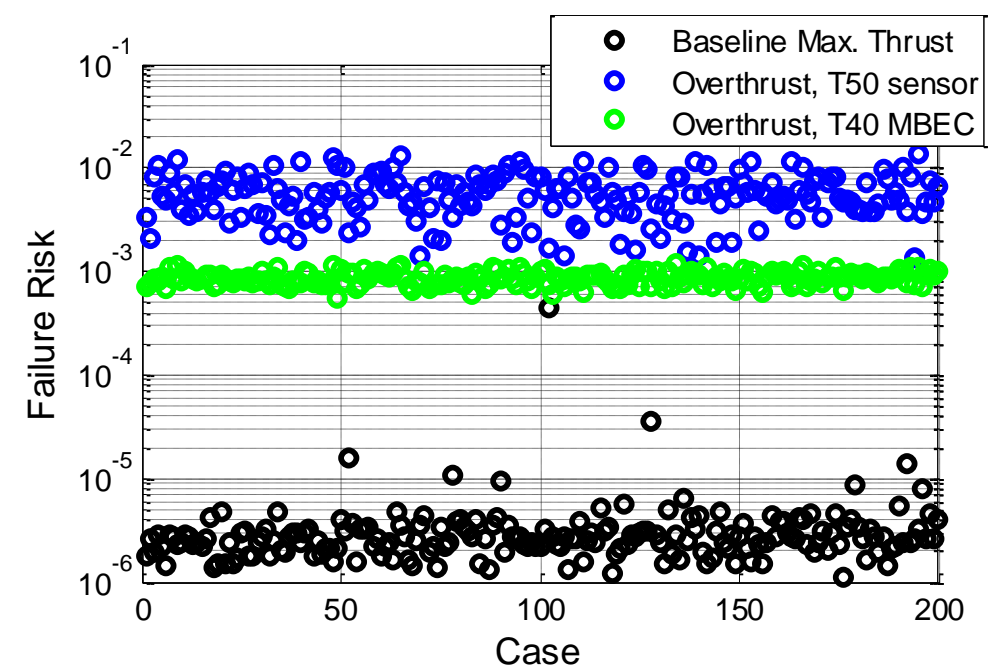

Figure 7 Calculated failure probability for 200 random cases operating at baseline maximum thrust, overthrust using the T50 sensor measurement to limit risk, and overthrust using the T40 measurement available with MBEC to limit risk.

Figure 8 reiterates these results in a different format. Here, the $10^{-3}$ risk level is plotted as the two-dimensional locus of allowable core speed and T40 values. As the figure shows, MBEC enables the overthrust mode to accurately and precisely push the engine to the desired risk level, whereas using the $T 50$ sensor measurements produces results that exceed the established risk boundary.

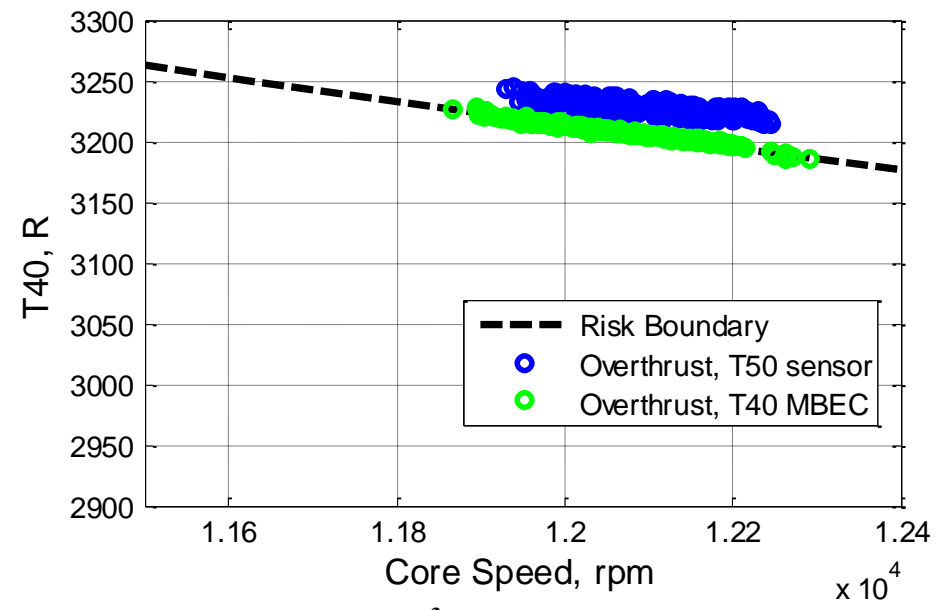

Figure 8 Turbine inlet temperature (T40) $10^{-3}$ risk level as a function of core speed (Risk Boundary)

\section{B. Faster Engine Response}

Previously-proposed controller modifications for the faster engine response control mode required retuning the EPR controller to increase the bandwidth and changing the acceleration schedule to allow the HPC to operate closer to the surge line. Additional modifications, to establish an operating mode referred to as high-speed idle, included changing the variable stator vanes and controller bandwidth to increase the fan speed while holding idle thrust constant, decreasing the efficiency of the engine, but allowing faster spool ups. The previous faster engine response work and risk analysis assumed a surge margin that represented the surge margin remaining after debits, such as tip clearance, deterioration, etc., were accounted for. Estimation of this signal is difficult because many of these debits are very nonlinear. Instead, the system here is designed to regulate the surge margin based on corrected flow and pressure ratio, without accounting for other debits. 


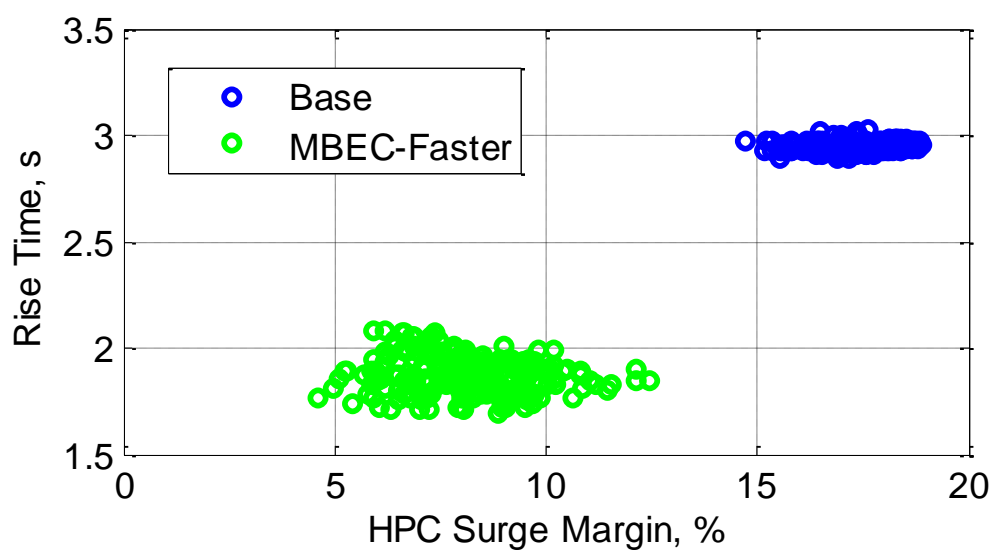

Figure 9 Relationship between rise time and minimum HPC SM for the baseline controller (CMAPSS40k) and MBEC with a reduced surge margin for emergency scenarios.

For the MBEC controller, the EEC incorporates two modification: a decrease in the surge margin limit and a slight increase in the controller bandwidth. In this example, the HPC SM value is changed to $7 \%$ (from 12\%), which approximates the same overall level of risk reported in Ref. 3. Figure 9 compares the relationship between the rise time and minimum surge margin for both the baseline engine and MBEC with a reduced HPC SM for emergency scenarios at the 200 test flight conditions.

Figure 9 shows that the surge margin controller allows the engine to operate at a slightly lower minimum surge margin (approximately 4.5\%) than intended (7\%) and produces a reduction in rise time, from 2.85- 3.05 seconds to 1.7-2.1 seconds. The error in the minimum surge margin can be attributed to both the accuracy of the estimated surge margin and the controller itself. These results could be improved by implementing more advanced controllers, or tuning the surge margin controller at more operating points.

\section{Discussion}

The MBEC architecture implemented in this work is an upgraded version of a previously-implemented MBEC architecture. ${ }^{8} \quad$ This MBEC featured a full envelope thrust controller, full envelope surge margin controller, and a high-pressure turbine inlet temperature controller. The OTKF used here adds two inputs, $P 25$ and $T 25$, to that in the previous work, which increases the accuracy of the on-board model and OTKF. This was shown, through simulation of throttle transients at 500 random takeoff conditions and 500 random cruise conditions, to be within $\pm 100 \mathrm{lb}$ thrust for over $99 \%$ of the tested conditions.

The MBEC architecture was modified to include an EEC that improves the capability of the engine through the generation of additional thrust, referred to as the overthrust control mode, or by allowing the engine to respond faster to the throttle command, referred to as faster engine response. For the overthrust control mode, to allow the engine to operate to higher power levels than originally designed for, the EEC modifies the set point command to allow the pilot to request up to $110 \%$ of the nominal thrust. Compared to previous emergency scenario work, the MBEC architecture offers two main improvements over the traditional engine controller when in the overthrust control mode. First, instead of extending and tracking the EPR of $N f$ commands, the thrust command range is increased and regulated. Second, instead of modeling the relationship between the high-pressure turbine inlet pressure and exhaust gas temperature, which is used to limit the risk, MBEC allows for the estimate of the HPT inlet temperature to be a feedback variable, which produced more accurate results than using the exhaust gas temperature.

The faster engine response control mode increases the performance of the engine by allowing it to respond more quickly to the throttle command. The main factor of concern in this control mode is compressor surge. Current techniques for protecting against compressor surge include regulating the corrected core acceleration, or the ratio of the fuel flow to combustor static discharge pressure. Both of these limiters are designed using a worst-case scenario, such as an end-of-life engine, which severely constrains the performance of newer engines. The MBEC architecture applied here estimates the surge margin, which allows newer engines to respond even faster and reach a lower surge margin during transient operation. 


\section{Summary}

This paper applies a model-based engine control (MBEC) architecture to the Commercial Modular Aero-Propulsion System Simulation 40,000 (CMAPSS40k) and demonstrates the advantage of using MBEC during emergency operation. This MBEC implementation contains an optimal tuner Kalman Filter (OTKF), which provides estimates of key unmeasured engine parameters, such as thrust, high-pressure turbine inlet temperature, and surge margin. Estimation of these parameters can impact the control system in several ways. The controller can deliver more consistent power to the airframe by estimating and directly regulating thrust. The control system can better avoid exceeding a critical physical limit which could severely decrease engine life, such as that imposed on the high-pressure turbine inlet temperature. By regulating an accurate estimate of the surge margin, the amount of extra margin required to be carried can be decreased. During an emergency flight scenario, sensed engine limits may be modified to allow the engine to perform better at an elevated risk of failure and to increase the overall safety of the aircraft. With the MBEC architecture, key engine limits that are not measured can be modified and directly used as feedback to the controller. Regulating these key engine parameters directly allows for better control over the amount of risk the engine is allowed to operate at and produces better performance for the assumed risk level.

\section{Acknowledgments}

The authors would like to thank Yuan Liu for his assistance in applying a risk based method to the MBEC architecture, the former Aviation Safety project for funding this work and the Transformative Tools and Technology project for continuing to advance research in model based engine control.

\section{References}

${ }^{1}$ Litt, J.S., Frederick, D.K., and Guo, T.-H., "The Case for Intelligent Propulsion Control for Fast Engine Response," AIAA 2009-1876, Infotech@ Aerospace Conference, Seattle, Washington, April 6-9, 2009.

${ }^{2}$ Csank, J.T, Chin, J.C., May, R.D., Litt, J.S., and Guo, T.-H., "Implementation of Enhanced Propulsion Control Modes for Emergency Flight Operation,” AIAA Infotech @ Aerospace Conference, St. Louis, MO, 29-31 March 2011.

${ }^{3}$ Liu, Y., Litt, J.S., and Guo, T.-H., "Design and Demonstration of Emergency Control Modes for Enhanced Engine Performance," AIAA 2013-3625, $49^{\text {th }}$ AIAA/ASME/SAE/ASEE Joint Propulsion Conference and Exhibit, San Jose, CA, July 14-17, 2013.

${ }^{4}$ Burcham, F.W., Jr, and Fullerton, C.G., —Controlling Crippled Aircraft - With Throttles, NASA TM-104238, 1991.

${ }^{5}$ Burcham, F.W., Jr., Fullerton, C.G., and Maine, T.A., - Manual Manipulation of Engine Throttles for Emergency Flight Control, NASA/TM-2004-212045, January 2004.

${ }^{6}$ May, R.D., Lemon, K.A., Csank, J.T., Litt, J.S., and Guo, T.-H., "The Effect of Faster Engine Response on the Lateral Directional Control of a Damaged Aircraft," AIAA 2011-6307, AIAA Guidance, Navigation, and Control Conference, Portland, OR, August 8-11, 2011.

${ }^{7}$ Litt, J.S, Sharp, L.M., and Guo, T.-H., "A Risk Assessment Architecture for Enhanced Engine Operation," AIAA Infotech@Aersopace Conference, Atlanta, GA, 20-22 April 2010.

${ }^{8}$ Connolly, J.W., Csank, J.T., Chicatelli, A.K., and Kilver, J., "Model-Based Control of a Nonlinear Aircraft Engine Simulation Using an Optimal Kalman Filter Approach," AIAA 2013-4002, 49 ${ }^{\text {th }}$ AIAA/ASME/SAE/ASEE Joint Propulsion Conference and Exhibit, San Jose, CA, July 14-17, 2013

${ }^{9}$ May, R.D., Csank, J.T., Lavelle, T.M., Litt, J.S., and Guo, T.-H., "A High-Fidelity Simulation of a Generic Commercial Aircraft Engine and Controller," AIAA 2010-6630, 46 ${ }^{\text {th }}$ AIAA/ASME/SAE/ASEE Joint Propulsion Conference and Exhibit, Nashville, TN, July 25-28, 2010.

${ }^{10}$ Csank, J.T., May, R.D., Litt, J.S., and Guo, T.-H., "Control Design for a Generic Commercial Aircraft Engine," AIAA 2010-6629, $46^{\text {th }}$ AIAA/ASME/SAE/ASEE Joint Propulsion Conference and Exhibit, Nashville, TN, July 2528, 2010.

${ }^{11}$ Richter, H. and Litt, J.S., "A Novel Controller for Gas Turbine Engines with Aggressive Limit Management," AIAA-2011-5857, 47th AIAA/ASME/SAE/ASEE Joint Propulsion Conference and Exhibit, San Diego, California, 31 July-3 August 2011

${ }^{12}$ Sharifi, R., Hacker, J., and Cao, C., "Adaptive Engine Control with Multiple Constraints," AIAA- 2011-1592, AIAA Infotech@Aerospace Conference, St. Louis, Missouri, 29-31 March 2011.

${ }^{13}$ Jaw, L., and Mattingly, J.D., Aircraft Engine Controls: Design, System Analysis, and Health Monitoring, American Institute of Aeronautics and Astronautics, Inc, VA, 2009.

${ }^{14}$ Simon, D.L., and Garg, S., "Optimal Tuner Selection for Kalman Filter-Based Aircraft Engine Performance Estimation," ASME, Journal of Gas Turbines and Power, Vol. 132, March, 2010. 
${ }^{15}$ Federal Aviation Administration, "Title 14 of the Code of Federal Regulations",

http://www.airweb.faa.gov/Regulatory_and_Guidance_Library/rgfar.nsf/MainFrame, accessed on May 2015 